\title{
Multiple Glomangiomas in a Patient With a History of Metastatic Melanoma
}

\author{
Sepehr Hamidi, MD; Gene H. Kim, MD; Brittney K. DeClerck, MD
}

\section{PRACTICE POINTS}

- The diagnosis of glomus tumor and glomangioma is easily suspected when the lesions are in the digital or subungual region.

- Multiple glomangiomas are rare and can clinically pose a diagnostic challenge to dermatologists.

- In patients with a recent history of malignancy, multiple glomangiomas may mimic cutaneous metastases. Therefore, multiple biopsies and histologic examination may be necessary.

To the Editor:

A 32-year-old man presented to the dermatology clinic with multiple asymptomatic blue lesions on the arms and upper torso of 15 years' duration. His medical history was notable for a recent diagnosis of malignant melanoma following excision of a mole on the upper back 4 months prior. He reported that the mole had been present since childhood, but his sister noticed that it increased in size and changed in color over the course of a year. Physical examination showed multiple blue subcutaneous nodules on the bilateral arms and lower back. The nodules were soft and nontender, and some had telangiectasia on the overlying skin.

Given the atypical distribution of nodules and the patient's recent history of melanoma, there was concern for cutaneous metastases. A punch biopsy of one of the nodules on the right upper arm was performed. Microscopic examination of the biopsy specimen revealed a proliferation of multiple cavernous vessels surrounded by several rows of monotonous round cells with moderate eosinophilic cytoplasm and monomorphic nuclei, which was consistent with a diagnosis of glomangioma (Figure 1). Immunohistochemical analysis showed diffuse positive staining for smooth muscle actin (Figure 2); CD34 immunostain was positive in endothelial cells and negative in tumor cells (Figure 3).

Two weeks after the first punch biopsy, the patient returned for follow-up. He noted a new soft, painless, nontender mass in the left axillary region. Positron emission tomography-computed tomography and a lymphoscintigram were performed to assess for lymphadenopathy, but they were not contributory. Subsequently, the patient underwent bilateral axillary sentinel lymph node dissection, which revealed the presence of metastatic melanoma in one lymph node in the left axilla. No metastatic disease was identified in the right axillary sentinel lymph nodes. A second skin biopsy was performed on another blue nodule to confirm the diagnosis and to exclude the possibility of sampling error. The histopathologic examination again revealed glomangioma, which established the diagnosis of multiple glomangiomas.

Glomus tumors arise from modified smooth muscle cells located in glomus bodies. The glomus body is a component of the dermis involved in regulation of body temperature that is composed of an afferent arteriole and an efferent venule. The arterial end of this apparatus, known as the Sucquet-Hoyer canal, is surrounded by glomus cells that have a contractile capability similar to smooth muscle cells. Glomus tumors usually present as

From the Department of Pathology, LAC+USC Medical Center and Keck University Hospital, University of Southern California, Los Angeles. Drs. Kim and DeClerck also are from the Department of Dermatology.

The authors report no conflict of interest.

Correspondence: Sepehr Hamidi, MD, Department of Pathology, David Geffen School of Medicine at UCLA, 10833 Le Conte Ave, Los Angeles, CA 90095 (shamidi@mednet.ucla.edu).

doi:10.12788/cutis.0136 

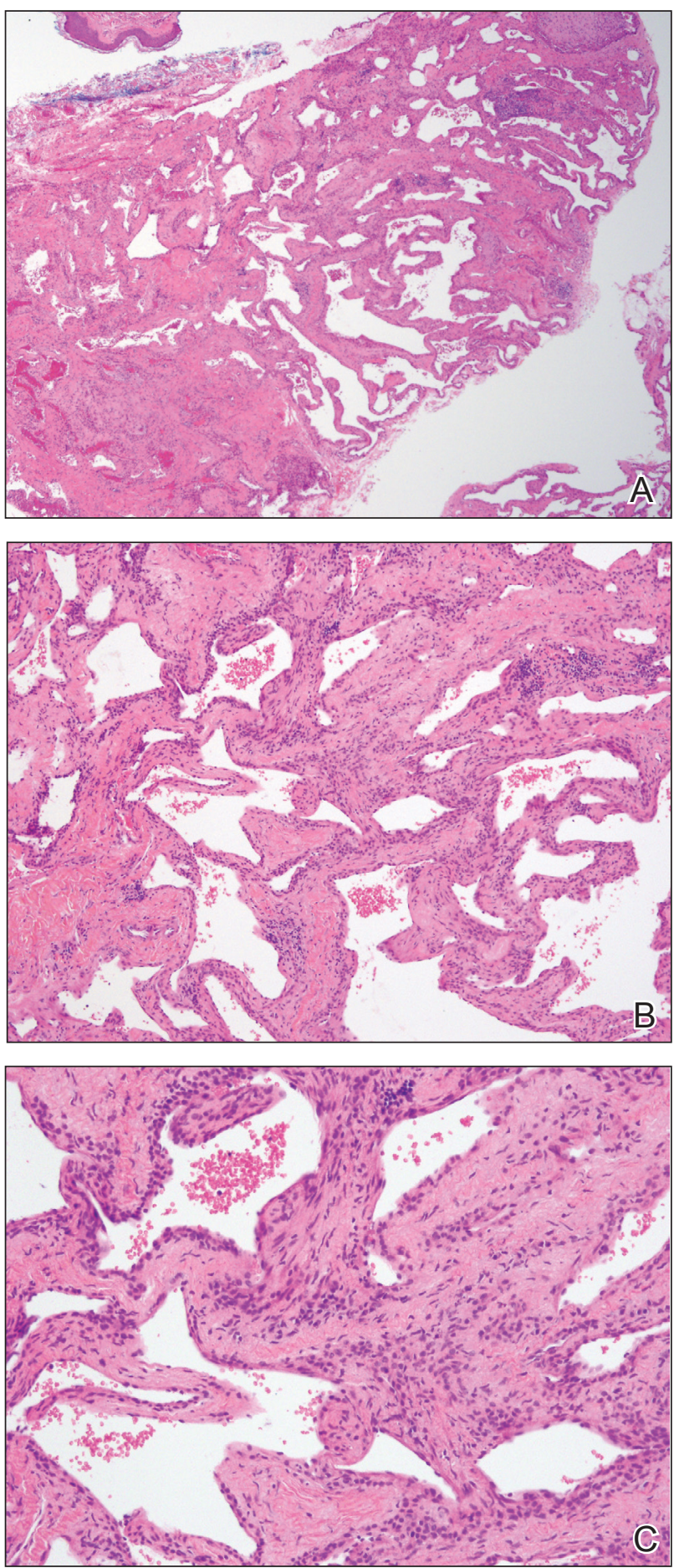

FIGURE 1. A, Several irregularly shaped, dilated vascular spaces in the dermis (H\&E, original magnification $\times 40$ ). B and $C$, Round uniform glomus cells surrounding the vascular channels were present $(\mathrm{H} \& \mathrm{E}$, original magnifications $\times 100$ and $\times 200$ ).

painful masses on the fingers with a typical subungual location and almost always are solitary. ${ }^{1}$ Glomangiomas, sometimes known as glomuvenous malformations, tend to be larger and usually are painless. They mostly are

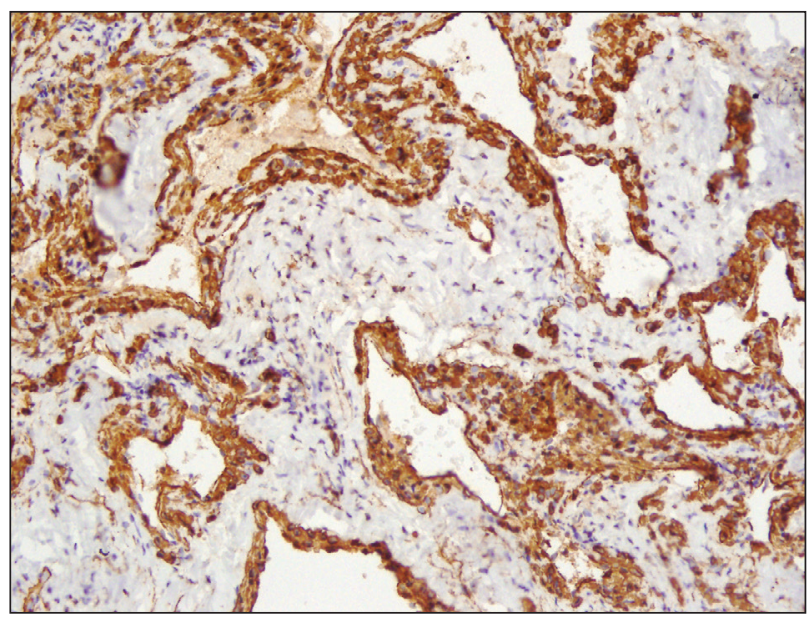

FIGURE 2. Immunohistochemistry revealed the glomus cells were positive for smooth muscle actin (original magnification $\times 200$ ).

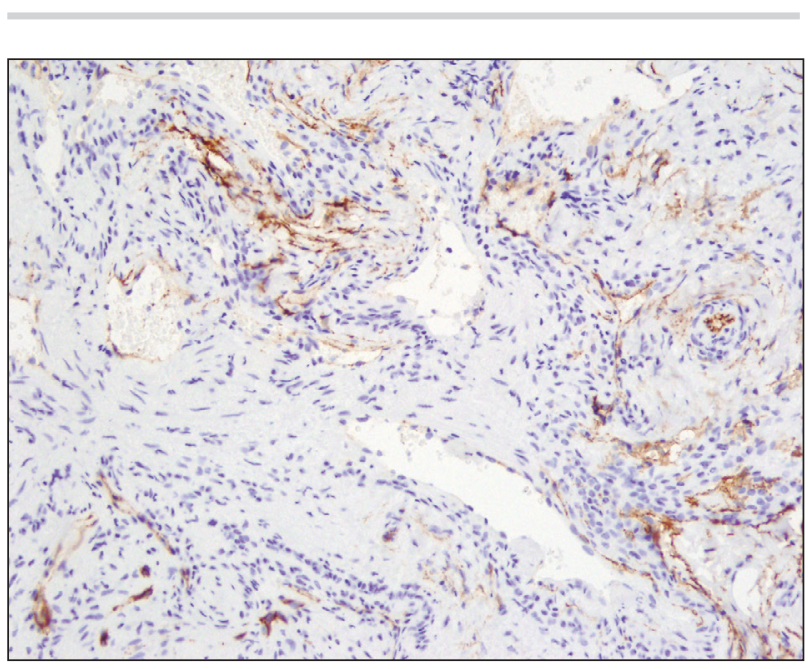

FIGURE 3. CD34 stain highlighted the endothelial cells (original magnification $\times 200$ ).

found on the trunk and extremities and can appear in groups. ${ }^{2,3}$ Histopathologically, glomus tumors are circumscribed lesions that show a predominance of glomus cells surrounding inconspicuous blood vessels. Glomangiomas are less well-circumscribed and show a more vascular architecture with prominent dilated vessels and a smaller number of glomus cells. ${ }^{4}$

We present a case of a patient with multiple glomangiomas. There are few reports of multiple glomangiomas in the literature. This case is particularly interesting in that our patient had a history of malignant melanoma, and there was a concern for skin metastases. Despite the patient's personal history of blue lesions that predated the diagnosis of melanoma for many years, we could not exclude the possibility of cutaneous metastases without performing biopsies.

Tumors of glomus cell origin usually are benign. It has been suggested to replace the term glomangioma with 
glomuvenous malformations to emphasize the hamartomatous nature of these lesions. ${ }^{5}$ Glomuvenous malformations, or glomangiomas, can occur sporadically or can be inherited as a familial disorder. Inheritable glomangioma has been linked to the chromosome 1p21-22 locus and mutations in the glomulin gene, GLMN, with variable penetrance. ${ }^{6}$ Our patient did not report a family history of such lesions.

Glomangiomas typically are solitary but rarely can present as multiple lesions in fewer than $10 \%$ of cases. ${ }^{7}$ Multiple glomangiomas are classified into 3 subtypes: localized, disseminated, and congenital plaque type. Localized multiple glomangiomas present as blue nodules confined to 1 anatomic location such as the hand or arm. Disseminated glomangiomas are more widely distributed and involve more than 1 anatomic location. ${ }^{8}$ Plaque-type glomangiomas consist of numerous confluent lesions occurring either as solitary or multiple plaques. ${ }^{2}$ Clinically, glomangiomas manifest as painless to mildly painful cutaneous nodules. Compared to venous malformations, glomangiomas are less compressible under external pressure.

Histopathologically, glomangiomas appear as nonencapsulated tumors with large, irregular, prominent vessels lined by glomus cells. Glomus cells may be so sparse that the distinction from venous malformations and hemangiomas becomes difficult. Immunohistochemistry can play an important role in diagnosis. As modified smooth muscle cells, glomus cells stain positive with $\alpha$-smooth muscle actin, while CD34 highlights the vascular endothelium.

The clinical differential diagnosis of multiple blue or violaceous subcutaneous nodules includes blue rubber bleb nevus syndrome, Maffucci syndrome, glomus tumor, pyogenic granuloma, hemangioma, spiradenoma, angiolipoma, leiomyoma, or hemangiopericytoma.9-12
Different treatment modalities are available for solitary glomangiomas, including surgical excision, sclerotherapy, and laser application. Treatment of multiple glomangiomas may not be feasible, and excision of isolated symptomatic lesions may be the only option; however, it is crucial to reach the correct diagnosis in these patients to avoid improper treatments and interventions.

\section{REFERENCES}

1. Patterson JW. Weedon's Skin Pathology. 4th ed. Edinburgh, Scotland: Churchill Livingstone Elsevier; 2016.

2. Mallory SB, Enjolras O, Boon LM, et al. Congenital plaque-type glomuvenous malformations presenting in childhood. Arch Dermatol. 2006;142:892-896.

3. Boon L, Mulliken JB, Enjolras O, et al. Glomuvenous malformation (glomangioma) and venous malformation distinct clinicopathologic and genetic entities. Arch Dermatol. 2004;140:971-976.

4. Gombos Z, Zhang PJ. Glomus tumor. Arch Pathol Lab Med. 2008;132:1448-1452.

5. Brouillard P, Boon LM, Mulliken JB, et al. Mutations in a novel factor, glomulin, are responsible for glomuvenous malformations ("glomangiomas"). Am J Hum Genet. 2002;70:866-874.

6. Brouillard P, Ghassibé M, Penington A, et al. Four common glomulin mutations cause two thirds of glomuvenous malformations ("familial glomangiomas"): evidence for a founder effect. J Med Genet. 2005;42:E13

7. Goodman TF, Abele DC. Multiple glomus tumors. a clinical and electron microscopic study. Arch Dermatol. 1971;103:11-23.

8. Miyamoto $\mathrm{H}$, Wada $\mathrm{H}$. Localized multiple glomangiomas on the foot. JDermatol. 2009;36:604-607.

9. Borovaya A, Kunte C, Flaig MJ, et al. Disseminated cutaneous glomangiomas in an adolescent boy. Acta Derm Venereol. 2012;92:324-325

10. Leger M, Patel U, Mandal R, et al. Glomangioma. Dermatol Online J. 2010;16:11.

11. Ertem D, Acar Y, Kotiloglu E, et al. Blue rubber bleb nevus syndrome. Pediatrics. 2001;107:418-420.

12. Faik A, Allali F, El Hassani S, et al. Maffucci's syndrome: a case report. Clin Rheumatol. 2006;25:88-91. 